\title{
Restoration of abraded and covered marks engraved in steel using active infrared thermography
}

\author{
by A. Quattrocchi*, S. A. Piccolo** and R. Montanini*** \\ *University of Messina, Dept. of Engineering, Contrada di Dio, Messina, Italy, aquattrocchi@unime.it \\ **Technical University of Denmark, Dept. of Management Engineering, Anker Engelunds Vej 1, Lyngby, \\ Denmark, sebpi@dtu.dk \\ ***University of Messina, Dept. of Engineering, Contrada di Dio, Messina, Italy, rmontanini@unime.it
}

\begin{abstract}
Alphanumeric marking is a common technique employed in industrial applications for identification of products. However, the realised mark can undergo deterioration, either by extensive use or voluntary deletion (e.g. removal of identification numbers of weapons or vehicles). For recovery of the lost data many destructive or non-destructive techniques have been endeavoured so far, which however present several restrictions. In this paper, active infrared thermography has been exploited for the first time in order to assess its effectiveness in restoring paint covered and abraded labels made by means of different manufacturing processes (laser, dot peen, impact, press and scribe). Optical excitation of the target surface has been achieved using pulse, lock-in and step heating. Raw infrared images were analysed with a dedicated image processing software originally developed in Matlab ${ }^{\mathrm{TM}}$, exploiting several methods, which include thermographic signal reconstruction, guided filtering and logarithmic transformation.
\end{abstract}

\section{Introduction}

In many technical applications, alphanumeric marking is used to identify, functionalize or simply decorate a target and to create an identification label, such is the case for serial number plates. Depending on the substrate material, operating condition and application, several manufacturing processes can be used for marking, which lead to different outcomes in terms of cost, quality and durability. Manual and pneumatic impacting, die stamping, mechanical punching, rolling, scribing, hot stamping, dot peen or stylus/pin marching are able to generate a mark through the mechanical deformation of the substrate material. Alternatively, processes that do not induce mechanical deformation of the base material can also be utilized, such as inkjet printing, chemical milling or laser sublimation [1-5].

When a metal is used, the marking force usually produces a permanent deformation of the base material. In particular, in the first substrate the mechanical process of engraving induces grains distortion, creating a plastic (permanent) deformation zone. In this region the basic properties of the material, such as hardness, electrical resistance, thermal conductivity and x-ray diffraction pattern, change. So, if this plastically deformed zone is still present after adulteration, it should be possible in principle to restore the label using either destructive or non-destructive methods. The amount of plastic flow and the depth to which the plastic region extends below the indentation, depend on the shape of the die and, as predictable, on the depth of indentation. If the plastic deformation region is not relievable, the recovering is very difficult and probably impossible [6]. Laser engraving is nowadays the most used laser marking technology on steel work pieces. It involves localized melting or vaporization of the surface with partial removing of the substrate material. In this case, permanent deformation does not occur or it is very limited, but the process creates an heat affected zone of few microns, instead [7].

With time, especially within an industrial or marine environment, marks can intentionally be covered with paint or they can undergo deterioration because of extensive use (e.g. wear) or corrosion. Moreover, in certain instances, such as for weapons or vehicle identification numbers, marks might be voluntarily deleted by criminals [8].

The recovery of alphanumeric tags is usually realized using either destructive or non-destructive methods [9]. They relies on the peculiar effect produced by the specific marking process used to fabricate the label.

Selective corrosion of acids [10] (e.g. chemical and electrochemical etching) is able to recover adulterated marks, especially if they have been originally engraved inducing consistent plastic deformation. The purpose of this process is to create a visible contrast between the damaged and undamaged regions of the substrate using the advantage that the damaged area usually has or has inducted a different electrochemical potential than the undamaged one. Heat treatment [11] is applied directly to the obliterated area until the metal glows in a light cherry red colour. This allows to release the residual tensile stresses and to bulge the deformed area by means of recrystallization effect. After the heating and the oxide layer removal, the recovered label is showed with a contrast to the dark surroundings. Ultrasonic cavitation [12] is another method used to obtained an erosion effect near the sites deformed by stamping.

Although destructive techniques are generally thought to give best results when compared to non-destructive ones, they cannot be repeated. This could represent a severe drawback in some instances, as in forensic investigations. For this reason, non-destructive methods might be more appropriate in these cases because of their possibility to be applied several times on the same target, without damaging it. NDT methods already exploited for recovering of 


\subsection{1/qirt.2016.103}

deteriorated marks include magnetoscopy [13] and X-rays [14]. Optical techniques, such as electron channelling contrast [15] and scanning acoustic microscope $[16,17]$, have been employed as well.

Active IR thermography is a powerful non-destructive technique that has been extensively used for detecting defects in materials $[18,19]$. However, it has never been applied to identify deteriorated alphanumeric marks.

This paper explores the feasibility of application of infrared thermography to recover paint covered and abraded marks engraved in steel by means of several manufacturing processes (laser, dot peen, impact, press and scribe). Optical excitation of the target surface has been achieved using pulse, lock-in and step heating. Raw infrared images were analysed with a dedicated image processing software originally developed in Matlab ${ }^{\mathrm{TM}}$, exploiting several methods, which include thermographic signal reconstruction, guided filtering and logarithmic transformation.

\section{$2 \quad$ Materials and methods}

\section{$2.1 \quad$ Engraved labels fabrication}

Five different samples were obtained by using the following fabrication techniques to engrave the base material (Fig. 1): laser sublimation (L), cold press (P), dot peen (D), impact (I) and scribe (S). The same base material (low carbon steel) was used for all the samples. In the following, some information about the employed marking techniques is given.

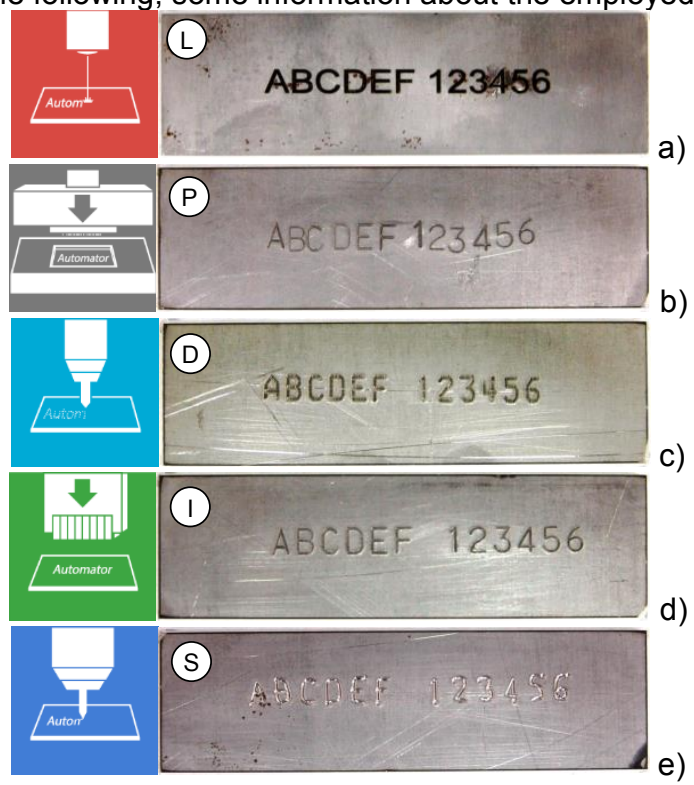

Fig. 1. Alphanumerical marks engraved in steel using different manufacturing techniques: a) laser sublimation $(L), b)$ cold press $(P), c)$ dot peen (D), d) impact (I) and e) scribe (S).

Laser marking $(\mathrm{L})$ was realized by sublimation of the target material by means of a fibre laser operated at $56 \mathrm{~W}$. Adopting this technique, tag style, dimension and depth could be adequately controlled with high accuracy.

Cold press marking $(P)$ worked a single letter at a time. The press was pneumatically activated with a fixed pressure. Such method did not allow characters depth and alignment to be guaranteed.

Dot peen (D) method consisted of a pneumatic peen controlled by an electronic system. The mark was created producing a sequence of ordinated dots to define a number or a letter, pushing and putting in vibration the machining pen onto the target surface. Such method did not allow characters depth to be guaranteed.

Impact marking (I) was obtained by means of a pneumatic unit equipped with a standard line typeholder. The method ensured uniform marking depth and perfect alignment of the characters, resulting in a high quality label.

Finally scribe marking $(S)$ employed a carbide tip that indents the surface of the metal. Being a manual technique, either characters size or alignment could not be guaranteed.

\begin{tabular}{lcccc}
\hline & Mean depth [mm] & $\mathrm{H} \times \mathrm{W}(\mathrm{Ar})$ letter "A" [mm] $\mathrm{H} \times \mathrm{W}(\mathrm{Ar})$ number "5" [mm] & $\mathrm{H} \times \mathrm{W}(\mathrm{Ar})$ number "6" [mm] \\
\hline Laser $(\mathrm{L})$ & $0.41 \pm 0.03$ & $4.66 \times 3.63(16.9)$ & $4.72 \times 3.48(16.4)$ & $4.75 \times 3.51(16.7)$ \\
\hline Press $(\mathrm{P})$ & $0.21 \pm 0.05$ & $5.11 \times 3.42(17.5)$ & $5.26 \times 3.60(18.9)$ & $5.24 \times 3.15(16.5)$ \\
\hline Dot peen (D) & $0.20 \pm 0.04$ & $5.41 \times 3.20(17.3)$ & $5.54 \times 3.05(16.9)$ & $5.46 \times 3.16(17.3)$ \\
\hline Impact (I) & $0.19 \pm 0.04$ & $5.17 \times 3.23(16.7)$ & $5.20 \times 3.70(19.2)$ & $5.25 \times 3.30(17.3)$ \\
\hline Scribe S) & $0.16 \pm 0.05$ & $5.64 \times 3.05(11.1)$ & $5.56 \times 3.23(18.0)$ & $5.70 \times 3.42(14.5)$ \\
\hline
\end{tabular}

Tab. 1. Measured depths and letter sizes $(H=$ height, $W=$ width, $A r=$ Area). 
After machining, each sample was carefully cleaned and analysed in order to check for the actual sizes and depths of the marks (Tab. 1). Sizes were measured by means of a calliper, while depths were retrieved using a laser triangulation sensor (MicroEpsilon $\mathrm{GmbH}$, mod. ILD 2200-20) mounted on an computer controlled micrometric translation stage (Phisic Instrumente Srl, mod. M-403.6DG).

\section{$2.2 \quad$ IR thermography methods}

The experimental setup consisted of a cooled infrared camera (Flir, mod. SC 7400), equipped with a $640 \times 512$ pixels InSb focal plane array detector working in the MWIR $(3.6-5.1 \mu \mathrm{m})$ spectral band (NETD $<20 \mathrm{mK}$ at ambient temperature), and a control and elaboration unit (Edevis $\mathrm{GmbH})$.

Heat deposition was achieved using either a ring flash of $10.8 \mathrm{~kJ}$ (pulse thermography, PT), or two halogen lamps of $1 \mathrm{~kW}$ each (lock-in thermography, LT and step heating thermography, SHT). PT was performed with an impulse duration of $10 \mathrm{~ms}$, recording a set of images for $1500 \mathrm{~ms}$ at $100 \mathrm{~Hz}$. SHT was carried out by heating the samples for 4 min and recording the cooling phase for $600 \mathrm{~s}$, with the same frame rate as before. A lock-in frequency of $0.1 \mathrm{~Hz}$ was used for LT. All experimental tests were carried out in reflection mode. The distance between the IR camera and the samples was chosen in order to obtain the best spatial resolution using a F/2 $50 \mathrm{~mm}$ lens. The same distance was maintained for all the applied stimulation methods to keep the spatial resolution of the IR image invariable.

\section{$2.3 \quad$ Testing procedures}

Controlled material removal was achieved at constant weight load using a lapping machine. Each sample was preliminary cut from the whole original plate and then englobed into a proper lapping jig. The procedure consisted in a pre-treatment of $60 \mathrm{~s}$ at $10 \mathrm{rpm}$ with wet P1200 grade abrasive sheet to eliminate dust and oxide from the surface, followed by successive time-controlled rounds at constant velocity with wet P80 grade abrasive sheet to remove progressively the base metal in six steps: $50 \mu \mathrm{m}, 100 \mu \mathrm{m} 150 \mu \mathrm{m}, 180 \mu \mathrm{m}$ and $200 \mu \mathrm{m}$. Final abrasion depths were obtained iteratively by measuring the reduced thickness with a micrometer gauge. Since the laser-made sample had an initial depth that is coarsely twice that of the other samples (Tab. 1), material removal was doubled for this sample $\left(L^{*}\right)$, so that the residual depth was approximately kept constant for all the samples.

A second set of samples was obtained by complete covering the "A" letter of each plate with black paint. A thickness of about $0.60 \pm 0.05 \mathrm{~mm}$ was finally obtained for all the examined samples. The testing parameter used for PT, LT and SHT were the same as for the abraded samples, except for the lock-in frequency that was $0.2 \mathrm{~Hz}$ for $\mathrm{D}, \mathrm{I}, \mathrm{P}$ and $\mathrm{S}$, and $0.4 \mathrm{~Hz}$ for $\mathrm{L}$.

\subsection{Image enhancement and filtering techniques}

All raw images were processed using the Matlab ${ }^{\mathrm{TM}}$ programming environment. Classical image enhancement techniques, such as histogram equalization, revealed to be of little help to reconstruct missing information.

Basically three approaches were used to post-process IR images: Thermographic Signal Reconstruction (TSR), developed by Shepard et al. and described in [20]; logarithmic transformation (LN), and the guided filter (GF), developed by $\mathrm{He}$ et al. and described in [21]. LN consists of computing the logarithm of each pixel of the image; this simple filter has interesting features because it allows the variance to be reduced, incrementing homoscedasticity. Guided filtering (GF) is an useful technique to perform a broad range of image processing tasks, including noise reduction, image enhancement, and flash denoising. For those reasons it appears to be a good candidate to approach the problem of thermographic image enhancement. In addition to those two methods, we applied a modified version of the guided filter: the original image has been divided into a number of blocks and for each block a guided filter for image enhancement is performed. With the application of the guided filter in a block (BGF) fashion we leveraged the local properties of the image. Regarding the application of the thermographic signal reconstruction, after the polynomial fitting to data, we used the first derivative images in the attempt to highlight the damaged region. Regarding the application of the guided filter for image enhancement, we computed the base layer applying the guided filter with parameters $r=8$ and eps $=0.1$; we computed the detail layer by subtracting the base layer from the original image; we boosted the detail layer by a factor 5 and combined it with the base layer. The same procedure (with $r=4$ and eps $=0.1$ ) was used for our block processing version of the guided filter, for each block.

\section{$3 \quad$ Results}

\subsection{Abraded marks}

Before IR thermography examination, each label has been photographed using a 50x digital microscope with integrated LED illumination source. Fig. 2 reports the obtained images for increasing abrasion depth. As it can be observed, in the visible range image quality is strongly affected by reflections caused by the translucent aspect of the surface and scratches generated by lapping. 


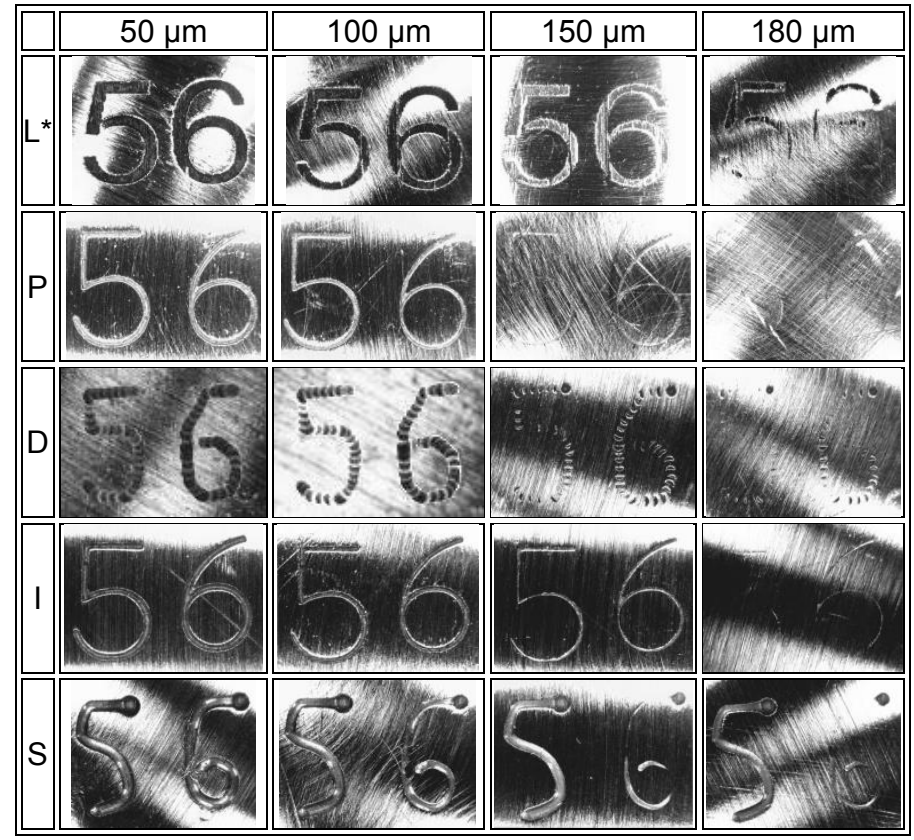

Fig. 2. Visible range (VR) images for increasing abrasion depth taken with a digital microscope (50X). Each raw corresponds to a specific marking technique: $L=l a s e r, P=$ press, $D=d o t$ peen, $I=i m p a c t, S=s c r i b e .{ }^{*}$ For the laser target the actual abrasion depth is twice that indicated in the first raw. (Images are not in scale)

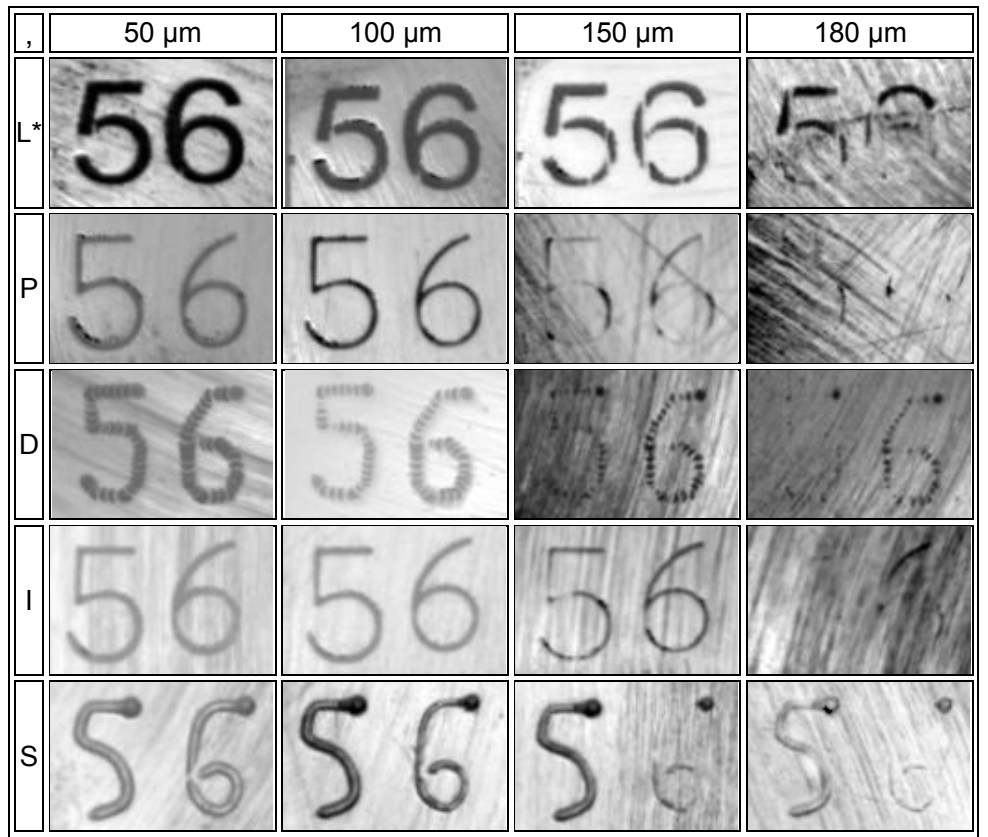

Fig. 3. Infrared images (IR) for increasing abrasion depth obtained after pulse thermography (PT). Each image has been selected qualitatively from the temporal sequence according to the "best appearance" criterium. Each raw corresponds to a specific marking technique: $L=l a s e r, P=$ press, $D=d o t$ peen, $I=i m p a c t, S=s c r i b e .{ }^{*}$ For the laser target the actual abrasion depth is twice that indicated in the first raw. (Images are not in scale)

Infrared images taken after pulse excitation (PT) are less affected by reflections instead (Fig. 3), resulting in superior contrast and enhanced readability. When comparing images in the visible range with those recorded in the MWIR range, one should remember that spatial resolution is quite different in the two cases, because of the different lens system adopted. In fact, while in the first case the resolution is $640 \times 480$ pixel, in the second one it is only $120 \times 80$ pixel. In addition, the depth of the characters is not always uniform, because of difficulties in controlling it, especially for cold press, dot peen and scribe making. Nevertheless, up to $150 \mu \mathrm{m}$ abrasion depth, both numbers are clearly recognizable, whatever marking technique is considered. At $180 \mu \mathrm{m}$ abrasion depth, the two fonts are still discernible for laser, dot peen and impact marking, whereas only one number can be distinguished for scribe marking. The worst 
situation occurred for cold press, were none of the numbers could be detected. Interestingly, the infrared pattern allowed the different depth of the engraving to be appreciated as well, see, for example, the number 5 in Fig. 3 , row $S$ or number 6 in Fig. 3, row D. Otherwise, the actual size of the font seems not to be as much important as the depth. However, although drawn definite conclusions from limited and not perfectly reproducible experimental data would be hazardous, it might guess that some distinctive features related to the marking technique can be emphasized. For example, laser marking resulted in a high thermal contrast due to the burned and well defined profile of the engraving, while cold press displayed poor thermal contrast at increased abrasion depth.

Fig. 4 shows a comparison between infrared images taken, after $200 \mu \mathrm{m}$ depth abrasion, with different excitation methods: pulse thermography (PT), lock-in thermography (LT) and step heating thermography (SHT).

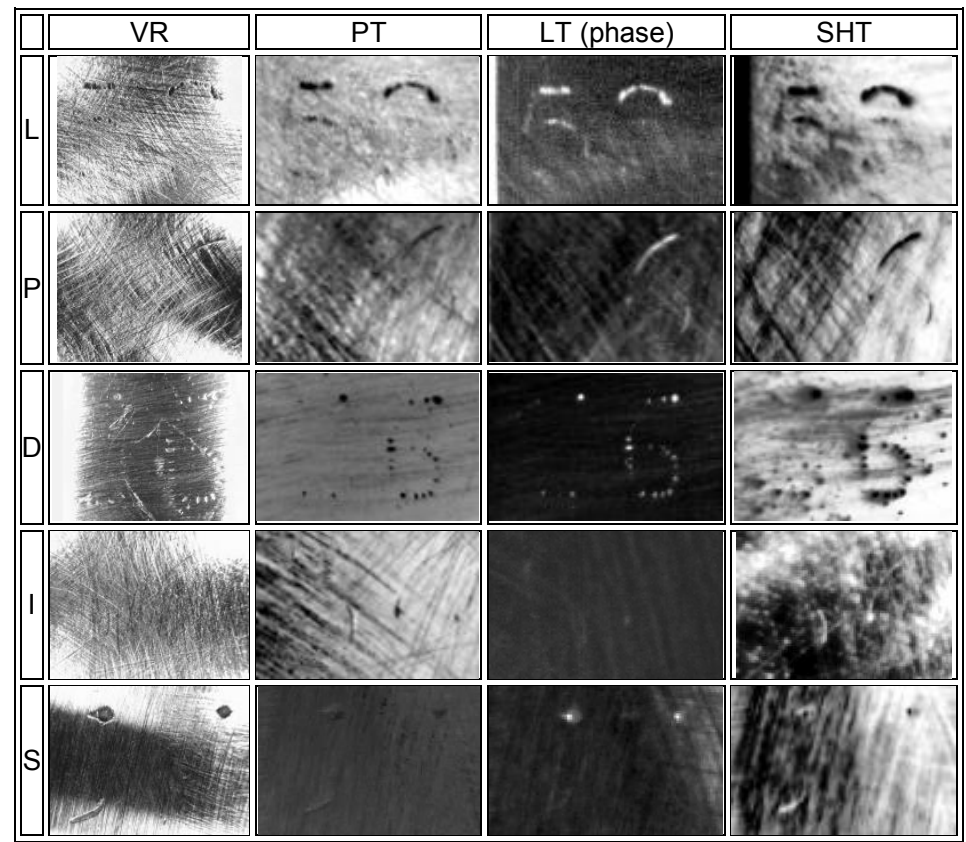

Fig. 4. Comparison between visible (VR) and infrared images (IR) obtained with pulse thermography (PT), lockin thermography (LT) and step heating thermography (SHT), after $200 \mu \mathrm{m}$ depth abrasion. (Images are not in scale)

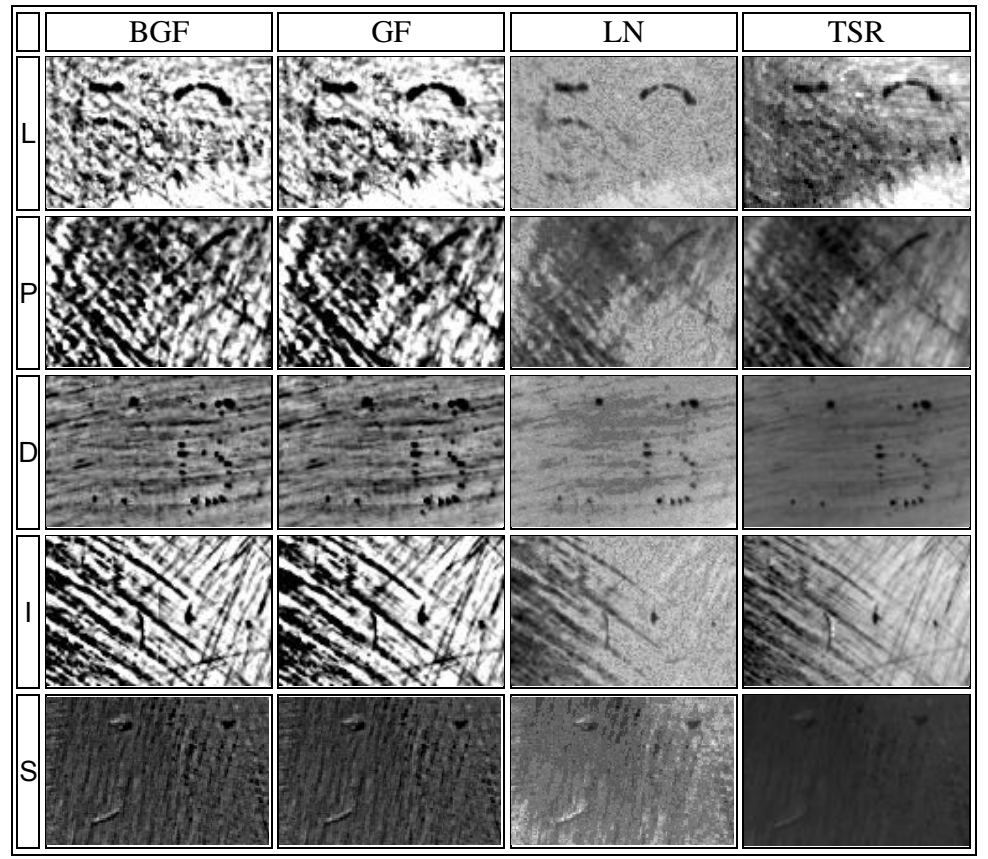

Fig. 5. Post-processed IR images obtained with pulse thermography (PT) after $200 \mu \mathrm{m}$ depth abrasion. BGF = Block Guided Filtering, GF = Guided Filtering, $L N=$ Logarithmic Transformation, TSR = Thermographic Signal Reconstruction, $1^{\text {st }}$ derivate. (Images are not in scale) 
Generally speaking, lock-in thermography gave the best outcome in terms of label identification. This is not surprising, seeing that LT phase imaging allows non uniform emissivity and Gaussian noise to be mitigated, resulting in high image quality, especially when L, D and P marking is considered. But, even with LT, no evident thermal signature remained after complete abrasion for I and S marking, whereas PT seems to return better result. In SHT consistent lateral heat diffusion occurred because of the higher heating duration, with limited benefits in label identification.

Fig. 5 shows post-processed IR images obtained by pulse thermography (PT), after $200 \mu \mathrm{m}$ depth abrasion. Both the guided and the block-guided filters resulted in an increase of the background noise, with minor effect deriving from block partition. In contrast, TSR and LN produced some image denoising. For L and D marking, for instance, LN is particularly effective in improving the contrast and the sharpness of the original PT infrared image.

TSR $-1^{\text {st }}$ derivative processing did not seem to provide relevant results for this application, probably because of the very limited thermal contrast of PT images.

We also post-process IR images recorded after lock-in (Fig. 6) and step heating (Fig. 7) excitation. In this case, three algorithms have been tested: Block Guided Filtering (BGF), Guided Filtering (GF), and Logarithmic Transformation (LN).

As far as LT phase images are concerned, the best results were obtained with BGF and GF, with little difference between the two methods. Both techniques are effective in increasing the thermal contrast, expressly for $D, L$ and $P$ marking. In this case, the intrinsic independency of the phase image from local optical and infrared features provided by LT helps in enhancing readability, which can be further improved by applying guided filtering.

In contrast, log transformation gave not as good results as in the case of PT.

Application of post-processing routines to SHT images showed some progress in handling cold press and dot peen labels. Otherwise, comments similar to those already made for the PT set of images can be drawn.

As a conclusive remark, it can be said that, independently from the technique used to manufacture the label, no information can be extracted thermally once the fonts have been completely erased. However, active thermography and proper image processing may provide themselves helpful in restoring partially erased labels which are awkward to be identified in the visible range.

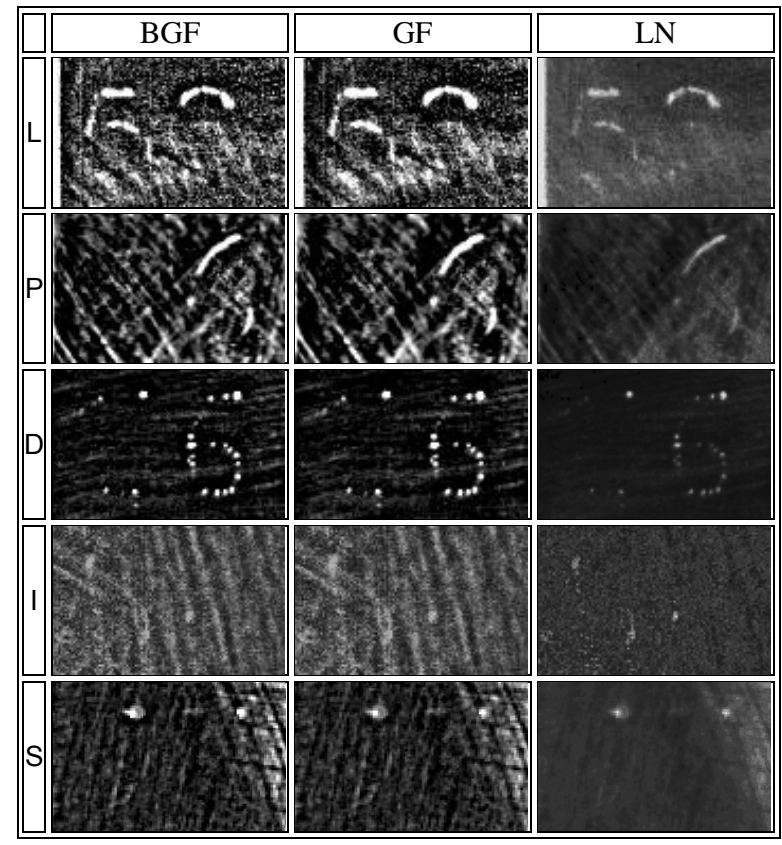

Fig. 6. Post-processed IR phase images obtained with lock-in thermography $(L T)$ after $200 \mu$ m depth abrasion. BGF $=$ Block

Guided Filtering, GF = Guided Filtering, $L N=$ Logarithmic

Transformation. (Images are not in scale)

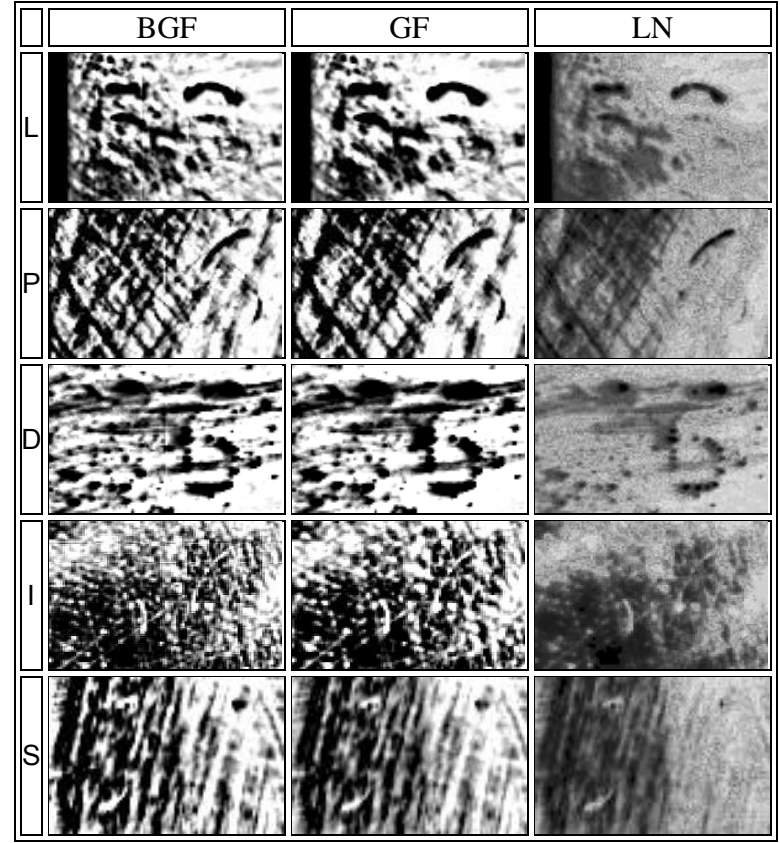

Fig. 7. Post-processed IR images obtained with step heating thermography (SHT) after $200 \mu$ m depth abrasion. $B G F=$ Block Guided Filtering, GF = Guided Filtering, $L N=$ Logarithmic Transformation. (Images are not in scale)

\subsection{Paint covered marks}

Fig. 8 shows the comparison between original visible image (VR), paint covered visible image (PVR) and infrared images obtained after pulse thermography (PT), lock-in thermography (LT) and step heating thermography (SHT).

Comparable outcomes were obtained with pulse and lock-in thermography, with the latter one providing better thermal contrast for all the examined targets. Step heating thermography instead never let to label identification.

Post-processed images are shown for PT, LT and SHT in Fig. 9, Fig. 10 and Fig. 11, respectively. 
For PT images, BGF and GF led to best font recover as L, P, D and I marking is considered. The font shape is correctly reproduced and the overall contrast with respect to the sound background enhanced. For $S$ marking, the identification of the underneath label is troublesome, and it not possible to discern the font.

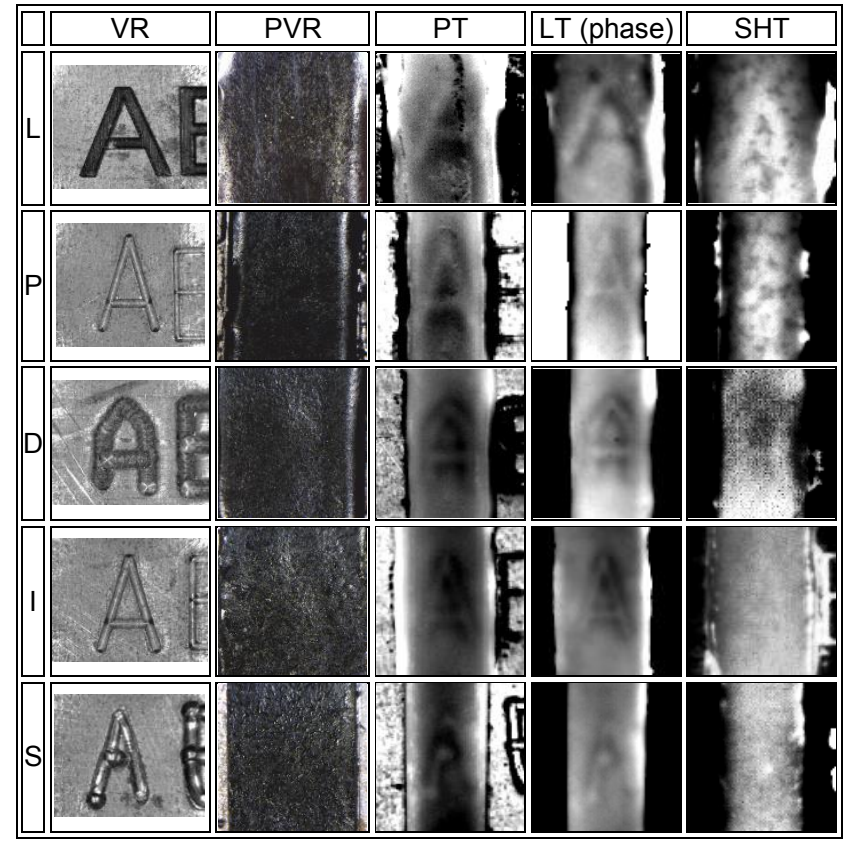

Fig. 8. Comparison between original visible range image (VR), paint covered visible range image (PVR) and infrared images obtained after pulse thermography (PT), lock-in thermography (LT) and step heating thermography (SHT). (Images are not in scale)

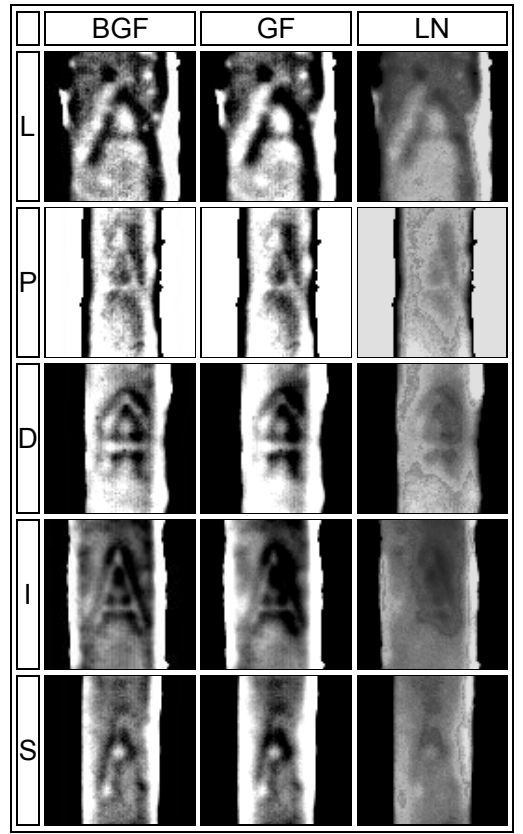

Fig. 10. Post-processed IR phase images obtained after lock-in thermography $(L T)$ on paint covered targets. BGF =

Block Guided Filtering, GF = Guided Filtering, $L N=$

Logarithmic Transformation. (Images are not in scale)

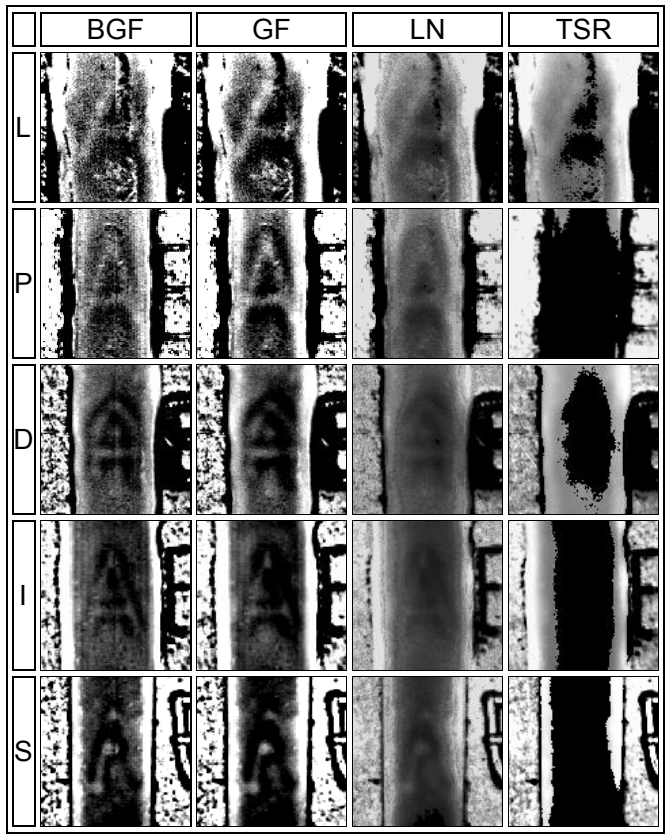

Fig. 9. Post-processed IR images obtained by pulse thermography $(P T)$ on paint covered targets. BGF = Block Guided Filtering, GF = Guided Filtering, $L N=\log$ Transformation, TSR = Thermographic Signal

Reconstruction, $1^{\text {st }}$ derivate. (Images are not in scale)

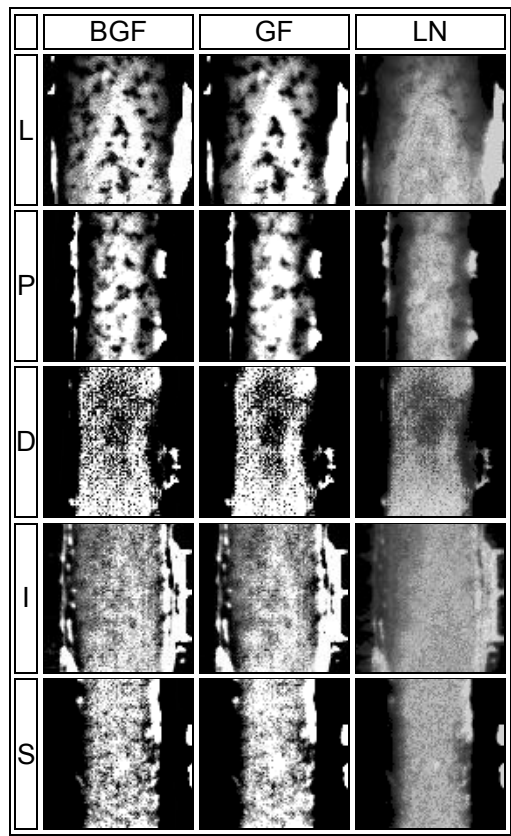

Fig. 11. Post-processed IR images obtained after step heating thermography (SHT) on paint covered targets. $B G F=$ Block Guided Filtering, $G F=$ Guided Filtering, $L N=$ Logarithmic Transformation. (Images are not in scale)

Logarithmic transformation is not so effective for paint covered labels, resulting in little or none improvement with respect to raw PT images. Even worst is the application of TSR, which hide the tag. 
Looking to results obtained on LT phase images, again a significant improvement can be highlighted by application of the BGF and GF techniques to all the examined font shapes, remarkably for those obtained with dot peen and scribe, while logarithmic transformation blends the font profile.

In contrast, as expected, application of image processing routines to IR images obtained after SHT did not lead to positive identification, although a partial recover of the letter $A$ in the impact and laser marking might be appreciated with the use of the guided filter.

\section{Conclusion}

Recovery of adulterated alphanumeric tags is of particular interest in several industrial fields. In forensic science, restoration of serial numbers of weapons or vehicles voluntary deleted by criminals is made more difficult by the advent of modern marking manufacturing techniques, such as laser sublimation. In addition, with time, especially within an industrial or marine environment, marks can intentionally be covered with paint or they can undergo deterioration because of extensive use (e.g. wear) or corrosion.

This paper explored the effectiveness of pulse, lock-in and step heating thermography in restoring covered and abraded marks obtained by laser, dot peen, impact, press and scribe marking on a steel surface. In particular, the limits of these IR techniques as well as the application of some filtering strategies to raw IR images have been investigated. Each manufacturing technique has specific influence in changing the properties of the substrate material. Moreover, the shape of the font is very different from one manufacturing technique to another one; this allowed the effect of font shape on thermal signature to be investigated as well.

All the active thermography techniques tested in this study proved to be helpful in reducing local optical reflection and disuniformity. Proper image processing of the raw images resulted in superior contrast and enhanced readability. In particular, good outcomes have been obtained by application of logaritmic transformation to raw PT images and by application of block guided filtering to raw phase lock-in images.

With pulse and lock-in thermography it is easy to recover label covered by paint, with the latter one providing better thermal contrast for all the examined targets. Step heating thermography instead never let to label identification.

\section{ACKOWLEDGMENTS}

The authors wish to thanks Eng. Claudio Buttà for his support in conducting the experimental tests.

The research was partially financed under the project POR SICILIA 2000-06 - Misura III.15 Az. C Progetto "Centro Ricerche Tecna Territorio" - PIT22 n.1999.IT.16.1.PO.011./3.15/5.2.13/0017.

\section{REFERENCES}

[1] Dillard Quinn F., Quinn A.W, "Apparatus and Method for Roll Forming and Marking Sheet Metal”, United States Patent, , no 5,009,093, 1991.

[2] Harrison M.E., Lee J., "Method for Optimizing Font Marking", United States Paten, no. 5,893,668, 1999.

[3] Calvert P., "Inkjet Printing for Materials and Devices", Chemistry of Materials, vol. 13, no. 10, pp. 3299-3305, 2001.

[4] Axtell III E.A., Kapp D.C., Knell T.A., Novotny M., Sakoske G.E., "Laser Marking Method and Apparatus", United States Patent, no. US 6,238,847 B1, 2001.

[5] Maiden N.R., "Serial Number Restoration: Firearm", pp. 1-4, Wiley Encyclopedia of Forensic Science, Australia, 2009.

[6] Pohl M, Katterwe H, Feyer M, Illenseer O., "Metallurgical Procedures for Detection of Deformations by Forensic Science Methods", Practical Metallography, vol. 26, pp. 405-413, 1995.

[7] Ion J., "Laser Processing of Engineering Materials: Principles, Procedure", pp. 385-394, Elsevier, 2005.

[8] Becker R.F., Dutelle A.W., "Criminal Investigation”, pp. 212-226, Burlington (Massachusetts), Jones \& Bartlett Learning, 2013.

[9] Katterwe H., "Restoration of Serial Numbers" in Stauffer E., Bonfanti M.S., "Forensic Investigation of StolenRecovered and Other Crime-Related Vehicles", pp. 177-205, Elsevier, Burlington, 2006.

[10] Zaili M.A.M., Kuppuswamy R., "Restoration of Engraved Marks on Steel Surfaces by Etching Technique", Forensic Science International, vol. 171, pp. 27-32, 2007.

[11] Feyer M, Pohl M, Katterwe H. "Restoration of Erased Numbers", Proceedings of the European meeting for shoeprint/toolmark examiners (SPTM 2001), Berlin (Germany), pp 23-31, 2001.

[12] Young S.G., "The Restoration of Obliterated Stamped Serial Numbers by Ultrasonically Induced Cavitation in Water", Twenty-ninth Semiannual Seminar of the California Association of Criminalists, Palo Alto (California), 1973.

[13] James S.H., Norbdy J.J., "Forensic Science: An Introduction to Scientific and Investigative Technique", Taylor \& Francis, 2014.

[14] Treptow R.S., "Handbook of Methods for the Restoration of Obliterated Serial Numbers", National Aeronautics and Space Administration, Cleveland (Ohio), 1978. 
[15] Ng B.C., Simkin B.A., Crimp M.A., Electron Channelling Contrast Imaging of Dislocation Structures in Deformed Stoichiometric NiAl, Materials Science and Engineering A, pp. 150-156. 1998.

[16] Quate C.F., The acoustic microscope, Scientific American, vol. 241, no. 9, pp. 58-66, 1979

[17] Da Silva L., Marques dos Santos P.A., "Recovering obliterated laser engraved serial numbers in firearms", Forensic Science International, vol. 179, pp. 63-66, 2008.

[18] Montanini R., "Quantitative determination of subsurface defects in a reference specimen made of Plexiglas by means of lock-in and pulse phase infrared thermography", Infrared Physics \& Technology, vol. 53, pp. 363-371, 2010.

[19] Montanini R., Freni F., "Non-destructive evaluation of thick glass fiber-reinforced composites by means of optically excited lock-in thermography", Composites: Part A, vol. 43, pp. 2075-2082, 2012.

[20] Shepard S.M., Lhota J.R., Rubadeux B.A., Wang D., Ahmed T., "Reconstruction and enhancement of active thermographic image sequences," Optical Engineering, vol . 42, no. 5, 2003.

[21] He K., Sun J. and Tang X., "Guided Image Filtering," IEEE Transactions on Pattern Analysis and Machine Intelligence, vol. 35, no. 6, pp. 1397-1409, 2013. 